\section{Empfehlung des ÄDA zur spezifischen Immuntherapie}

D er Ärzteverband Deutscher Allergologen setzt sich intensiv dafür ein, dass die spezifische Immuntherapie als indikationsbezogene Besonderheit in die Anlage 3 der Musterempfehlung zur Richtgrößenvereinbarung aufgenommen wird. In diesem Zusammenhang führte der Präsident des Verbandes, Herr Dr. Josef Wenning, mehrere Gespräche mit Herrn Professor Brech, Mitglied des Ausschusses der Ärzte und Krankenkassen sowie Mitglied der Arzneimittelkommission.

Herr Professor Brech hat in diesen Gesprächen zugesagt, dass er sich für die Aufnahme der Hyposensibilisierungstherapie in die Anlage 3 einsetzen wird. Ein kurzfristiges Erreichen dieses Zieles hält er auf Bundesebene jedoch kurzfri- stig für wenig wahrscheinlich, da in der Arzneimittelkommission zur Zeit eine Blockadesituation bestehe, die von den Beratern der Spitzenverbände der Krankenkassen ausgehe.

\section{Aufnahme in regionale Vereinbarungen empfohlen}

Er empfiehlt daher dem Ärzteverband Deutscher Allergologen mit Hilfe der regionalen Vertretungen auf regionaler Ebene mit den kassenärztlichen Vereinigungen und Krankenkassen eine solche Lösung anzustreben.

In Brandenburg wurde die Aufnahme der spezifischen Immuntherapie in die Anlage 3 der Arzneimittelrichtgrößen-Vereinbarung bereits verwirklicht.
Bis zu einer besseren Lösung empfiehlt Herr Professor Brech den allergologisch tätigen Ärzten folgendes Vorgehen:

Bei der Rezeptur einer spezifischen Immuntherapie sollten die Patientendaten, die Indikation und die Rezeptur in eine Liste aufgenommen werden und diese Liste sollte zusammen mit der Quartalsabrechnung unter Hinweis auf die Praxisbesonderheit der Immuntherapie den Abrechnungsunterlagen beigefügt werden.

Der Ärzteverband Deutscher Allergologen empfiehlt zusätzlich den Hinweis, dass die spezifische Immuntherapie qualitätsgesichert entsprechend dem Positionspapier der WHO durchgeführt wurde und dass der Praxisinhaber durch die Facharztweiterbildung und / oder durch die Zusatzbezeichnung Allergologie über die notwendigen allergologischen Kenntnisse, Fähigkeiten und Erfahrung verfügt.

J. Wenning

\title{
IGeL für Allergologen
}

\section{Der Katalog der „Individuellen Gesundheitsleistungen“, kurz IGeL- Liste genannt, ist nun zwei Jahre alt. Am 10. Mai 2000 formulierte der Ärzteverband Deutscher Allergologen (ÄDA) in Bad Godesberg mit Dr. L. Krimmel den aktuellen Stand der IGeL-Leistungen für aller- gologisch tätige Ärzte.}

Eolgende allergologische Leistungen können nicht mit der Krankenkasse, aber direkt mit den Patienten abgerechnet werden:

- Allergie-Check (Präventionsuntersuchung): Klinische Untersuchung, Hauttest, Gesamt-IgE, RAST, Eosinophile, ECP, Basophile, Sekretzytologie auf Wunsch ohne Vorliegen einer allergieverdächtigen Anamnese und Symptomatik

- Pulmo-Check (Präventionsuntersuchung): Klinische Untersuchung der Lunge, Lungenfunktion, Sputumuntersuchung, ggf. Röntgen-Thorax auf Wunsch bei symptomfreier Anamnese

- Allergologische Berufseingangsuntersuchung: Je nach geplantem Beruf klinische Untersuchung der beanspruchten Organe, Hauttest, Lungenfunktion, ggf. mit Provokation unspezifischer Substanzen (z.B. Azetylcholin), Gesamt-IgE, RAST, ECP, SekretUntersuchungen und Beratung

- Wehrtauglichkeit: Über das übliche Maß der Allergiediagnostik hinausgehende Untersuchungen wie Belastungs-, Provokationstests und Invitro-Untersuchungen sowie schriftliche Beurteilung

- Analyse von Allergenen aus Materialproben der Patienten: z.B. Milben, Schimmelpilze

- Hauttests und In-vitro-Tests ohne Anamnese und ohne klinischen Befund: z.B. Amalgam und Quecksil- ber, Nahrungsmittel bei Pollenallergie ohne klinische Symptome einer Nahrungsmittelallergie (klinisch stumme Sensibilisierungen)

- Hausbesuch zur Ermittlung von Allergenen im Wohnbereich

- Gutachterliche Leistungen und Bescheinigungen auf Wunsch des Patienten

- Überprüfung des Immunstatus ohne anamnestische Hinweise auf Immundefekt auf Wunsch des Patienten: Hauttest auf Recall-Antigene, Immunglobuline, Komplementfaktoren, Zellzablanalysen im Blut

- Tests zur Verträglichkeit von Kosmetika: Prick-, Epikutan-, Provokations- und In-vitro-Tests

Bei weitergehenden Fragen können Sie sich an Herrn Dr. Krimmel, MedWell Gesundheits-AG, Im Winkel 1,

D-51145 Köln wenden.

Tel.: $02203 / 295634$

Fax: $02203 / 23819$

E-Mail: info@medwell.de

Internet: www.medwell.de

J. Sennekamp 